\title{
Results of specialization in the surgical treatment of gastric cancer in Peru
}

\author{
Michel Portanova, Fernando Vargas, Emilio lombardi, Victor Mena, Ramiro Carbajal, Nestor Palacios, \\ and Jorge OrRego \\ Gastric Cancer Service, Department of General Surgery, Rebagliati National Hospital, Lima, Peru
}

\begin{abstract}
Background. The best results in the surgical treatment of gastric cancer are those obtained by the Japanese surgical school that emphasizes D2 lymphadenectomy as a fundamental principle for obtaining better local control of the disease. However, this technique has not gained wide acceptance in the West, owing to the fact that the results of Japanese studies have not been reproduced frequently in Western countries. In recent years, a series of studies have recommended the centralization of gastric cancer treatment in specialized surgical units in order to obtain results similar to those achieved by Japanese centers. The objective of this study was to describe the specialization process and to show the short-term results obtained in the surgical treatment of gastric cancer in the Specialized Unit of the Rebagliati National Hospital, the largest general referral hospital in Lima, Peru.

Methods. In the year 2000 a specialized service was created for the surgical treatment of gastric cancer, initiating a process that included the establishment of surgical treatment guidelines, training in the Japanese surgical technique, and progress along the learning curve for D2 lymphadenectomy. Clinical, surgical, and pathological data were recorded prospectively in a fixed format, considering that strict documentation of cases was also an important step within this process.

Results. Between January 1, 2004, and December 31, 2005, 243 consecutive patients with a proven diagnosis of gastric adenocarcinoma were admitted to the operating theater for surgical treatment. During this study period, morbidity was $\mathbf{2 2 . 7} \%$ and hospital mortality, $2.8 \%$. The numbers (mean \pm SD) of resected lymph nodes for distal gastrectomy and total gastrectomy were $37.3 \pm 12.4$ and $45.3 \pm 14.5$, respectively. Hospital stay was 13 days for distal gastrectomy as well as for total gastrectomy.

Conclusion. According to our results, adequate training in the Japanese surgical technique, progress along the learning curve for D2 lymphadenectomy, and the establishment of special-
\end{abstract}

Offprint requests to: M. Portanova

Calle Guatemala 165 - Dpto. 101, Urb. Santa Patricia, La Molina, Lima, Perú

Received: August 29, 2006 / Accepted: February 4, 2007 ized units are highly recommended for the surgical treatment of gastric cancer in Western referral hospitals.

Key words Gastric cancer · Gastrectomy · Lymphadenectomy $\cdot$ Specialization

\section{Introduction}

Gastric cancer is the second most frequent cause of death from cancer in the world. It reaches its highest incidence in countries in East Asia, for example, Japan [1]. South America has also recorded a significant incidence, making it a high-risk area; and in Peru in particular, it is the leading cause of cancer-related death [2]. Surgical treatment has been recognized as the cornerstone of gastric cancer management, and the Japanese surgical school, with its classic lymphadenectomies, has obtained the best short- and long-term results [3,4]. However, the use of their techniques is not widespread in the West, partially as the result of two important studies conducted in Europe, in which D1 and D2 lymphadenectomies were compared and no benefits were found regarding survival. On the contrary, D2 lymphadenectomy was found to be associated with greater morbidity and mortality $[5,6]$. Nevertheless, these studies have been questioned, owing to the lack of experience and mastery of the technique on the part of the participating surgical teams, among other reasons [7].

On the other hand, Western centers where emphasis is placed on specialization, progress along the learning curve, hospital volume, and surgeon volume are now obtaining results similar to those reported by Japanese centers $[8,9]$. For this reason, the centralization of patients in specialized units for the surgical treatment of gastric cancer is highly recommended in the West [1012]. The present study describes the experience and short-term results of the surgical treatment of patients 
with gastric cancer at the specialized unit of the Rebagliati National Hospital, the largest national referral hospital in Peru.

\section{Patients and methods}

In the year 2000, the Surgery Department of the Rebagliati National Hospital decided to form teams specializing in oncological pathology, thereby creating a gastric cancer service and centralizing the surgical treatment of patients affected by this disease. At that time a dynamic process was initiated in this specialized service, which included the following steps: (1) the formation of a surgical team; (2) the establishment of treatment guidelines and protocols in accordance with the recommendations of the Japanese Gastric Cancer Association, in order to standardize patient management; (3) training and improvement of our compliance with the Japanese surgical technique, including a training program at the National Cancer Center in Tokyo; (4) progress along the learning curve for D2 lymphadenectomy; (5) rigorous documentation of cases for auditing, follow-up, and investigational purposes, in which the clinical, surgical, and pathological data were recorded prospectively in a fixed format; and (6) multidisciplinary integration.

Between January 1, 2004, and December 31, 2005, 266 consecutive patients with a histopathological diagnosis of gastric neoplasia were admitted to our specialized surgical service; 243 (91.3\%) of them had a proven diagnosis of adenocarcinoma and they were the patients included in this study. Patients with other diagnoses were excluded.

Preoperative studies were routinely performed by using upper endoscopy, upper gastrointestinal barium meal, chest X-ray, and abdominal computed tomography (CT) scan. Afterward, the patients were admitted to the operating theater for a laparoscopy. In patients with peritoneal or hepatic metastasis, only peritoneal lavage for cytological examination was performed, and biopsy samples from peritoneal or hepatic nodules were obtained. In the absence of peritoneal or hepatic metastasis, the patients were immediately submitted to a laparotomy to attempt curative surgery, following peritoneal lavage for cytological examination. Laparoscopy was omitted in patients with early-stage cancer, and in patients with advanced-stage cancer who had obstruction or bleeding.

The standard surgical procedure was gastrectomy associated with D2 lymphadenectomy, in accordance with the guidelines established by the Japanese Gastric Cancer Association [13,14]; however, some patients were submitted to a D1 lymphadenectomy owing to their overall deteriorated physical condition and significant associated comorbidity.
The morbidity reported in this study was classified as either surgical complications (e.g., leakage, abscess) or medical complications (e.g., pneumonia, urinary tract infection). Hospital mortality was defined as postoperative death within 30 days, or death within the same hospitalization. Morbidity and mortality refer exclusively to patients with resective surgery.

All analyses in the present study were based on the prospective data collected in our specialized service.

\section{Results}

Of the 243 patients with a diagnosis of adenocarcinoma, $59.2 \%$ were male (144/243) and $40.8 \%$, female (99/243); the mean age was 66.7 years (range, 27-95 years).

In 67 patients (28\%), resective surgical treatment was not possible and they only had bypass surgery, exploratory laparotomy, or laparoscopy.

The remaining 176 patients (72\%) had resective surgery. The mean age of this group of patients was 66.9 years (range, 27-89 years); $57.9 \%$ were male (102/176) and $42.1 \%$ were female (74/176). Patient demographics and tumor characteristics are presented in Table 1. Ninety-five patients had distal gastrectomy, 75 had total gastrectomy, 2 had proximal gastrectomy, 2 had pylorus-preserving gastrectomy, and 2 had resection of the gastric remnant. Splenectomy was performed in 36 patients.

Table 1. Patient demographics and operations in the resective surgery group

\begin{tabular}{lc}
\hline Sex & \\
Male & 102 \\
Female & 74 \\
Male-female ratio & $1.3: 1$ \\
Age (years) & 66.9 \\
Mean & $27-89$ \\
Range & \\
Tumor location & 28 \\
Upper 1/3 & 68 \\
Middle 1/3 & 80 \\
Lower 1/3 & \\
T stage (pathologic) & 28 \\
T1 & 87 \\
T2 & 60 \\
T3 & 1 \\
T4 & \\
Histological type & 93 \\
Differentiated & 83 \\
Undifferentiated & \\
Operations & 95 \\
Distal gastrectomy & 75 \\
Total gastrectomy & 2 \\
Proximal gastrectomy & 2 \\
Pylorus-preserving gastrectomy & 2 \\
Gastric remnant resection & \\
\hline
\end{tabular}


Table 2. Operative morbidity and mortality in patients with resective surgery

\begin{tabular}{lcc}
\hline & No. of patients & $\%$ \\
& 176 & 100 \\
\hline Complications & 40 & 22.7 \\
Surgical & 31 & 17.6 \\
Pancreatic leakage & 8 & 4.5 \\
Pancreatitis & 4 & 2.2 \\
Esophageal leakage & 5 & 2.8 \\
Duodenal leakage & 1 & 0.5 \\
Biliary leakage & 1 & 0.5 \\
Colonic leakage & 1 & 0.5 \\
Stenosis or obstruction & 5 & 2.8 \\
Wound infection & 3 & 1.7 \\
Wound dehiscence & 2 & 1.1 \\
Intraperitoneal bleeding & 2 & 1.1 \\
Abscess & 2 & 1.1 \\
Ileus, dumping & 1 & 0.5 \\
Medical & 9 & 5.1 \\
Pneumonia & 5 & 2.8 \\
Pleural effusion & 1 & 0.5 \\
Tracheobronchitis & 1 & 0.5 \\
Renal failure & 2 & 1.1 \\
Urinary tract infection & 1 & 0.5 \\
Hospital mortality & 5 & 2.8 \\
\hline
\end{tabular}

Of the total of 95 patients who underwent a distal gastrectomy, 83.1\% (79/95) had a D2 lymphadenectomy. Of the total of 75 patients who underwent a total gastrectomy, $81.3 \%(61 / 75)$ had a D2 lymphadenectomy.

In the patients who underwent a distal gastrectomy with D2 lymphadenectomy, the number of lymph nodes retrieved (mean $\pm \mathrm{SD}$ ) was $37.3 \pm 12.4$ (range, 20-87); while in patients who underwent a total gastrectomy with D2 lymphadenectomy, the number of lymph nodes retrieved (mean \pm SD) was $45.3 \pm 14.5$ (range, 22-87).

The incidence of morbidity in patients with resective surgery was $22.7 \%$, surgical complications occurred in $17.6 \%$ of the patients, and medical complications occurred in $5.1 \%$. Pancreatic complications occurred in $6.7 \%$ of the patients, and intestinal leakage (esophageal, colonic, duodenal) in $3.8 \%$. In terms of the medical complications, respiratory diseases were predominant (Table 2).

Hospital mortality for patients with resection was $2.8 \%(5 / 176)$; the causes of death are listed in Table 3. All the patients who died were over 70 years old.

Hospital stay was 13 days for distal as well as for total gastrectomies.

\section{Discussion}

Surgical resection continues to be the only potentially curative treatment for gastric cancer, and the best results are those reported by the Japanese surgical school
Table 3. Causes of death in patients with resective surgery

\begin{tabular}{lcl}
\hline Operation & $\begin{array}{c}\text { Age } \\
\text { (years) }\end{array}$ & Cause of death \\
\hline Distal gastrectomy & 72 & Bleeding \\
Distal gastrectomy & 76 & Hepatic trauma \\
Distal gastrectomy $_{\text {Total gastrectomy }}^{\mathrm{a}}$ & 79 & Abscess \\
Total gastrectomy $^{\mathrm{a}}$ & 73 & Abscess \\
\hline
\end{tabular}

${ }^{\mathrm{a}}$ Splenectomy was included

$[3,4]$. Despite this, D2 lymphadenectomy has not been widely accepted in the West, partially because of the results of prospective studies conducted in Europe which found no difference in survival rates between D1 and D2 lymphadenectomies, while, on the contrary, the D2 was found to be related to greater morbidity and mortality [5,6]. However, these studies have been seriously questioned because the surgeons were not familiar with the technique, they had not reached the plateau of the learning curve for D2 lymphadenectomy, and the studies also included too many hospitals, among other reasons [7,11].

In contrast, a new randomized prospective study conducted by the Italian Gastric Cancer Group, comparing D1 and D2 lymphadenectomies, has shown short-term results that were totally different, and comparable to those obtained by Japanese studies [8], generating expectation about the long-term results. The methodology used by the Italian group to conduct this study provides an encouraging model. They included only five hospitals, and in each one they chose only two surgeons with proven experience in D2 lymphadenectomy. Additionally, this prospective study was preceded by a phase II study to evaluate the safety and effectiveness of D2 lymphadenectomy, including only two surgeons for each of nine participating hospitals. A supervising surgeon who had had training in Japan was appointed, and adequate quality control was established for surgical procedures. The short- and long-term results obtained in this phase II study were comparable to those for Japanese studies [9].

It is important to note that, if evidence-based medicine is discussed, the Italian study which compared D1 and D2 corresponds to the same evidence level as the two previously mentioned European studies. Therefore, it is now possible to state categorically that the D2 is not associated with greater morbidity or mortality when performed at very experienced centers or by very experienced surgeons.

In their prospective study comparing D2 with extended paraaortic lymphadenectomy (study 9501, headed by Sano [4]), the Japan Clinical Oncology Group (JCOG) established adequate quality control including surgeons with experience of more than 100 D2 gastrec- 
tomies or institutions with a specialized unit that had an annual gastrectomy volume of 80 cases or more. In the same way, in the JCOG study 9502, headed by Sasako [15], surgeons with experience of more than 100 D2 gastrectomies or institutions with a specialized unit that did at least 50 gastrectomies a year were selected for a randomized controlled trial comparing the left thoracoabdominal approach versus the abdominaltranshiatal approach for gastric cancer of the cardia or subcardia; both studies indicated that experience, technical skill, hospital volume, and surgeon volume were relevant in managing highly complex surgical procedures.

A detailed analysis by Sasako [10] of the surgical treatment of gastric cancer highlights the relation between the average annual number of gastrectomies and short-term results. Furthermore, recent studies conducted in the West also show the importance of hospital volume and surgeon volume, indicating that both factors are directly related to the best results in the short term $[16,17]$. For example, it has been reported that mortality decreased significantly in hospitals performing more than 63 gastrectomies per year [18]. For gastric cancer, the operative mortality rate decreased by $41 \%$ for each increase of ten patients in surgeons' annual surgical caseloads [19]. Thus, it now clearly appears that hospital volume and surgeon volume are critical factors that determine the morbidity and mortality in highly complex surgery [20].

The concept of subspecialization in surgery is also backed by a series of Western studies, such as the study by Callahan et al. [21], in which it was concluded that the adjusted mortality risk was substantially lower when gastrectomies were performed by trained subspecialized surgeons. Additionally, Western centers that have adopted working systems of this type and have recognized the learning curve for the D2 lymphadenectomy have shown great improvement in their results, which are similar to those of the Japanese studies; for example, Lewis et al. [22] found that surgical subspecialization reduced operative mortality from $12 \%$ to $3.1 \%$ over a period of 5 years.

Taking into account the recommendations for centralization, as well as the results achieved by specialized groups in the West, and recognizing the influence of surgeon and hospital volume; a group of surgeons highly motivated in the management of gastric cancer were recruited into a specialized surgical team at the Rebagliati National Hospital, the largest general referral hospital in Peru. That executive decision constituted a challenging new experience for our country, in which, for decades, general surgeons have managed all types of intraabdominal pathology. This important step was taken in order to provide the best conditions under which to treat patients with gastric cancer and to obtain short- and long-term results comparable to those of the most important hospitals in the world.

Surgical treatment guidelines were established immediately in order to standardize all the procedures. These guidelines are based on the recommendations of the Japanese Gastric Cancer Association. This step is fundamental for performing serious work as a team and for unifying criteria in the selection of treatment, thus improving mutual understanding with the diverse centers that perform gastric cancer surgery according to the guidelines of the Japanese school [13].

Taking into account that the D2 lymphadenectomy corresponds to the Japanese technique, we believe that direct experience at its very source is an important step that a gastric cancer surgical team should consider relevant in order to implement a working system such as that of the Rebagliati National Hospital, not only because it enables surgeons to learn technical and surgical details from an "in-situ" experience, but also because it permits them to see the manner in which surgical treatment is linked to other specialties involved in the management of this pathology, and in general, to become familiar with the fundamentals and working philosophy of the Japanese school. For this reason, part of our surgical team has received training at the National Cancer Center in Tokyo as an important part of our strategic plan.

The learning curve for D2 lymphadenectomy is reported to plateau after $15-30$ procedures [23,24]. The centralization of cases in our specialized service has also permitted each surgeon on our team to progress rapidly along the learning curve for D2 lymphadenectomy, which is a fundamental step for performing this type of surgery optimally, because this is a technically demanding procedure. In addition, centralization is important for investigative purposes [12], as it permits close, rigorous documentation of all cases, which are managed under the same guidelines.

Regarding the analysis of our results, the operative mortality of $2.8 \%$ in our study is within the range reported for the most important studies. For example; in Japan, mortality between $0.4 \%$ and $2.7 \%$ was reported [3]; Degiuli and the Italian Gastric Cancer Group [8,25] report mortality between $0.6 \%$ and $3.1 \%$; Martin et al. [26], from the Memorial Sloan Kettering Cancer Center in the United States, report a 3.6\% mortality, and Siewert et al. [27], in Germany, report a mortality rate of $5.1 \%$. In our study, all the patients who died were over 70 years old. In this regard, some studies report higher mortality in elderly patients, especially depending on associated comorbidity and the functional reserve of their organs [28]. In our patient who died of hepatic trauma (an uncommon cause of death), the trauma was related to a rough maneuver with the hepatic retractor. The patient underwent relaparotomy because of a large 
Table 4. Comparative short-term results of two different working systems at Rebagliati National Hospital

\begin{tabular}{lccc}
\hline & Mortality (\%) & Morbidity (\%) & $\begin{array}{c}\text { No. of resected } \\
\text { lymph nodes } \\
\text { (range) }\end{array}$ \\
\hline Seven General Surgery Services 1996-1999 & 10.9 & 31.5 & $11-20$ \\
Specialized Gastric Cancer Unit 2004-2005 & 2.8 & 22.7 & $20-87$ \\
\hline
\end{tabular}

hepatic hematoma and an uncontrolable intraabdominal hemorrhage.

Our morbidity of $22.7 \%$ is also comparable to that in the most important series. In Japan, morbidity rates of between $6.9 \%$ and $30.3 \%$ are reported [3]; Degiuli et al. [8,25] report morbidity rates of $13.6 \%$ to $20.9 \%$, and Siewert et al. [27] report a morbidity rate of $29 \%$. Our most frequent surgical complication was related to the pancreas (pancreatic leakage, $4.5 \%$; pancreatitis, $2.2 \%$ ). Regarding this, of the 12 patients with pancreatic complications, none had a pancreatic tail resection. In 3 of these 12 patients, a total gastrectomy with splenectomy was performed according to the Maruyama technique; in 1 patient, a total gastrectomy without splenectomy was performed, and the remaining 8 patients had a distal gastrectomy without splenectomy. That is, most of our patients with pancreatic complications had no splenectomy, and our explanation for this outcome is that D2 lymphadenectomy by itself, as is known, demands constant dissection near the pancreatic tissue.

In our study, esophageal leakage occurred in $6.3 \%$ of the 79 patients in whom esophageal anastomosis was performed. The 5 patients who developed an esophageal leak had a two-layer, hand-sewn, end-to-side anastomosis. Furthermore, it is important to note that 4 cases occurred in 2004 ( 3 of them during the first semester) and only 1 case occurred during 2005. We believe that the tendency towards a decrease in the incidence of this complication is related not only to our gained experience but also to the use of staples to perform esophageal anastomosis.

It should be noted that none of the five patients with esophageal leakage died, which we attribute to the experience gained by our surgical team in the management of serious complications. The experience of the surgical team in the management of adverse effects of treatment is another factor that has been highlighted [10]; therefore, it is very important that it be taken into account.

Quality control in D2 lymphadenectomy has long been based on the number of lymph nodes resected. In the present study, we obtained a mean of 37 lymph nodes for distal gastrectomy and 45 for total gastrectomy. However, the most important series report their results without differentiating the type of gastrectomy performed. In this sense, our overall mean number of resected lymph nodes was 41 , a good mean if we compare it to the results in the following reports: Sano et al. [4], in Japan, reported a mean number of 54; Siewert et al. [27], in Germany, reported 45; Wu et al. [29], in China, reported 37; and, Degiuli et al. [8], in Italy, reported 36 .

It is important to note that the results achieved in the present study regarding morbidity, mortality, and the number of lymph nodes retrieved surpassed those reported by our hospital when there was no specialized unit [30] (Table 4). We believe that the improvement in our results is due primary to a change in the working system, with a specialized surgical unit, because other factors, such as intensive and anesthetic care, antimicrobial therapy, and artificial nutritional therapy have been always of high quality, because our hospital is the largest and most important national referral center in Perú.

Therefore, the establishment of a specialized unit for the surgical treatment of gastric cancer has constituted the most significant advance in recent years in the management of gastric cancer, not only at our hospital, but in our country. Part of our vision as a specialized surgical team is based on the words of Sano and colleagues [31], in their report about 1000 consecutive gastrectomies without operative mortality: "We believe that zero mortality is a monument to technical specialization".

In the West, where the incidence of gastric cancer is lower than that in Japan and, therefore, opportunities for training and surgical development are also lower in comparison with opportunities in Japan, we highly recommend the centralization of gastric cancer treatment in specialized surgical units, with adequately trained surgeons who have completed the learning curve for D2 lymphadenectomy, and who follow adequate treatment guidelines, in order to obtain results comparable to those attained in the Japanese studies.

\section{References}

1. Parkin DM, Bray F, Ferlay J, Pisani P. Global cancer statistics, 2002. Cancer J Clin 2005;55:74-108.

2. Torres R. The prioritization of gastric cancer in Perú. Work document. Epidemiology General Office. Lima: Peruvian Ministry of Health; 2001. p. 31-2. 
3. Fujii M, Sasaki J, Nakajima T. State of the art in the treatment of gastric cancer: from the 71st Japanese Gastric Cancer Congress. Gastric Cancer 1999;2:151-7.

4. Sano T, Sasako M, Yamamoto S, Nashimoto A, Kurita A, Hiratsuka M, et al. Gastric cancer surgery: morbidity and mortality results from a prospective randomized controlled trial comparing D2 and extended para-aortic lymphadenectomy — Japan Clinical Oncology Group Study 9501. J Clin Oncol 2004;22:2767-73.

5. Cuschieri A, Weeden S, Fielding J, Bancewicz J, Craven J, Joypaul V, et al. Patient survival after D1 and D2 resection for gastric cancer: long term results of the MRC randomized surgical trial. Br J Cancer 1999;79:1522-30.

6. Bonenkamp JJ, Hermans J, Sasako M, Van de Velde CJH. Extended lymph-node dissection for gastric cancer. N Engl J Med 1999;340:908-14.

7. Sasako M. Principles of surgical treatment for curable gastric cancer. J Clin Oncol 2003;23:274-5.

8. Degiuli M, Sasako M, Calgaro M, Garino M, Rebecchi F, Mineccia M, et al. Morbidity and mortality after D1 and D2 gastrectomy for cancer: interim analysis of the Italian Gastric Cancer Study Group randomized surgical trial. Eur J Surg Oncol 2004;30: 303-8.

9. Degiuli M, Sasako M, Ponti A, Calvo F. Survival results of a multicentre phase II study to evaluate D2 gastrectomy for gastric cancer. Br J Cancer 2004;90:1727-32.

10. Sasako M. Role of surgery in multidisciplinary treatment for solid cancers. Int J Clin Oncol 2004:9:346-51.

11. Spanknebel K, Brennan M. Is D2 lymphadenectomy for gastric cancer a staging tool or a therapeutic intervention? Surg Oncol N Am 2002;11:415-30.

12. Hartgrink H, Van de Velde C. Status of extended lymph node dissection: locoregional control is the only way to survive gastric cancer. J Surg Oncol 2005;90:153-65.

13. Nakajima T. Gastric cancer treatment guidelines in Japan. Gastric Cancer 2002;5:1-5.

14. Japanese Gastric Cancer Association. Japanese classification of gastric carcinoma; second English edition. Gastric Cancer 1998;1: $10-24$.

15. Sasako M, Sano T, Yamamoto S, Sairenji M, Arai R, Kinoshita $\mathrm{T}$, et al. Left thoracoabdominal approach versus abdominaltranshiatal approach for gastric cancer of the cardia or subcardia: a randomised controlled trial. Lancet Oncol 2006;7:644-51.

16. Birkmeyer J, Siewers A, Finlayson E, Stukel TA, Lucas FL, Batista I, et al. Hospital volume and surgical mortality in the United States. N Engl J Med 2002;346:1128-37.

17. Biffi R, Chiappa A, Luca F, Pozzi S, Lo Faso F, Cenciarclli S, et al. Extended lymph node dissection without routine splenopancreatectomy for treatment of gastric cancer: low morbidity and mortality rates in a single center series of 250 patients. J Surg Oncol 2006;93:394-400.

18. Hannan H, Radzyner M, Rubin D, Dougherty J, Brennan MF. The influence of hospital and surgeon volumes on in-hospital mortality for colectomy, gastrectomy, and lung lobectomy in patients with cancer. Surgery 2002;131:6-15.

19. Bachmann M, Alderson D, Edwards D, Wotton S, Bedford C, Peters TJ, et al. Cohort study in south and west England of the influence of specialization on the management and outcome of patients with oesophageal and gastric cancer. Br J Surg 2002; 89:914-22.

20. Mansfield P. Lymphadenectomy for gastric cancer. J Clin Oncol 2004;22:2759-61.

21. Callahan M, Christos P, Gold H, Mushlin AI, Daly JM. Influence of surgical subspecialty training on in-hospital mortality for gastrectomy and colectomy patients. Ann Surg 2003;238:629-36.

22. Lewis W, Edwards P, Barry J, Khan S, Dhariwal D, Hodzovic I, et al. D2 or not D2? The gastrectomy question. Gastric Cancer 2002;5:29-34.

23. Parikh D, Johnson M, Chagla L, Lowe D, McCulloch P. D2 gastrectomy: lessons from a prospective audit of the learning curve. Br J Surg 1996;83:1595-9.

24. McCulloch P. D1 versus D2 dissection for gastric cancer (letter) Lancet 1995;345:1516-7.

25. Degiuli M, Sasako M, Ponti A, Soldati T, Danese F, Calvo F. Morbidity and mortality after D2 gastrectomy for gastric cancer: results of the Italian Gastric Cancer Study Group prospective multicenter surgical study. J Clin Oncol 1998;16:1490-3.

26. Martin R, Jaques D, Brennan M, Karpeh M. Extended local resection for advanced gastric cancer. Increased survival versus increased morbidity. Ann Surg 2002;236:159-65.

27. Siewert RJ, Bottcher K, Stein H, Roder JD. Relevant prognostic factors in gastric cancer: 10-year results of the German Gastric Cancer Study. Ann Surg 1998;228:449-61.

28. Hara H, Isozaki H, Nomura E, Fujii K, Sako S, Tanigawa N. Evaluation of treatment strategies for gastric cancer in the elderly according to the number of abnormal parameters on preoperative examination. Jpn J Surg 1999;29:837-41.

29. Wu CW, Hsiung CA, Lo SS, Hsich MC, Shia LT, Whang-Peng J, et al. Randomized clinical trial of morbidity after D1 and D3 surgery for gastric cancer. Br J Surg 2004;91:283-7.

30. Flores-Cabral JA, Vojvodic I, Ortega D, Lombardi E. Factors associated with postoperative mortality following gastric adenocarcinoma surgery at the Edgardo Rebagliati Martins Hospital. Rev Gastroenterol Peru 2004;24:212-22.

31. Sano T, Katai H, Sasako M, Maruyama K. One thousand consecutive gastrectomies without operative mortality. Br J Surg 2002; 89:123. 